\title{
Impact of early diagnosis on functional preservation in patients with rheumatoid arthritis: the early bird catches the worm
}

\author{
Yun-Hong Cheon and Sang-Il Lee
}

Divison of Rheumatology, Department of Internal Medicine and Institute of Health Science, Gyeongsang National University School of Medicine, Jinju, Korea

Received: June 19, 2017

Accepted: June 22, 2017

\section{Correspondence to}

Sang-Il Lee, M.D.

Divison of Rheumatology, Department of Internal Medicine and Institute of Health Science, Gyeongsang National University School of Medicine, 79 Gangnam-ro, Jinju 52727, Korea Tel: +82-55-750-8853

Fax: +82-55-758-9122

E-mail: goldgu@gnu.ac.kr

\section{See Article on Page 738-746}

Rheumatoid arthritis (RA) is a chronic inflammatory disease that causes severe joint pain, stiffness, and irreversible damage, as well as physical, social, and psychological functional impairment. These characteristics of RA result in decreased quality of life (QOL) and premature mortality [1]. Previous studies have demonstrated that early diagnosis and administration of disease-modifying anti-rheumatic drugs (DMARDs, synthetic or biologic) lead to less joint damage and better physical function. Therefore, over the past 20 years, the paradigm of RA diagnosis and treatment has changed to prevent radiological progression and functional impairment [2-4].

Keeping pace with this paradigm shift, the purpose of RA treatment has changed to improve health-related function as well as to prevent joint destruction [5]. The two different aspects of health-related function are general health condition and RA-specific health condition [6]. The Health Assessment Questionnaire-Disability Index (HAQ-DI) has been used to investigate RA-specific health function, but it does not evaluate social or psychological aspects, such as anxiety and depression, which affect QOL. It contains 20 questions, with scores ranging from 0 to 3 . A score of zero indicates no functional disability [7].

Several studies have evaluated the effect of early diagnosis on functional outcome in patients with RA [4,8-10]. Nell et al. [4] confirmed that patients with very early RA (median disease duration, 3 months) showed greater functional improvement after 3 months. Gremese et al. [10] also demonstrated that patients with symptom duration of $\leq 3$ months achieved an HAQ-DI score $<0.5$ after 1 year compared with patients with symptom duration $>3$ months. However, no study has been conducted in Korean patients with RA.

In the current issue of the Korean Journal of Internal Medicine, Kim et al. [11] reported a cross-sectional study examining the effect of early diagnosis and treatment on functional status in patients with RA of various disease durations. They enrolled 4,540 patients with RA and divided them into early diagnosis (lag time between symptom onset and diagnosis $<12$ months) and delayed diagnosis (lag time $>12$ months) groups to evaluate the impact of early diagnosis on the development of functional disability using the HAQDI. Throughout the study, patients in the early diagnosis group had low- 
er HAQ-DI scores than those in the delayed diagnosis group ( $0.64 \pm 0.63$ vs. $0.70 \pm 0.66, p<0.01$ ). Early diagnosis (odds ratio [OR], 1.19; 95\% confidence interval [CI], 1.01 to 1.40), male sex (OR, 2.88; 95\% CI, 2.29 to 3.63), disease duration < 5 years (OR, 1.42; 95\% CI, 1.16 to 1.75), higher education level (OR, 1.70; 95\% CI, 1.39 to 2.07), absence of bone erosion at diagnosis (OR, 1.42; 95\% CI, 1.20 to 1.68), age (OR, $0.98 ; 95 \% \mathrm{CI}, 0.97$ to 0.99 ), and disease activity score 28 -erythrocyte sedimentation rate (OR, 0.45; $95 \% \mathrm{CI}, 0.97$ to 0.99 ) were independently associated with lower functional disability. In addition, they investigated the influence of early diagnosis on functional disability according to disease duration and revealed that early diagnosis was associated with no functional disability in patients with disease duration < 5 years (OR, 1.37; 95\% CI, 1.09 to 1.72), but not in patients with a longer disease duration (5 to 10 years; OR, 1.07; 95\% CI, 0.75 to 1.52). These findings confirm that an early diagnosis within 1 year is associated with a lower risk of functional disability for at least 5 years.

The results of the study by Kim et al. [11] are consistent with those of previous studies [4,8-10]. Furthermore, this study is meaningful because it is the first to include Korean patients with RA to confirm the effect of early diagnosis on functional outcome using a large, nationwide, observational cohort. Other advantages of this study are its relatively large sample size, population-based design, and inclusion of extensive demographic information. These advantages allow for adjustment of several known risk factors, such as age, sex, education and bone erosion, and for direct analysis of the effect of early diagnosis on functional outcome based on disease duration. However, because this study was cross-sectional, recall bias cannot be excluded. Another limitation is that the authors did not re-evaluate the HAQ-DI score; therefore, the effect of synthetic or biologic DMARDs on improvement or deterioration of functional outcome could not be analyzed.

In conclusion, considering these previous studies, early diagnosis of RA is helpful in preventing major radiographic progression and delaying development of a functional disability, particularly in patients with a shorter disease duration. A future prospective cohort study should be conducted to confirm the impact of early diagnosis on functional outcome in patients with RA.

\section{Conflict of interest}

No potential conflict of interest relevant to this article was reported.

\section{REFERENCES}

1. Smolen JS, Aletaha D, McInnes IB. Rheumatoid arthritis. Lancet 2016;388:2023-2038.

2. Aletaha D, Neogi T, Silman AJ, et al. 2010 Rheumatoid arthritis classification criteria: an American College of Rheumatology/European League Against Rheumatism collaborative initiative. Arthritis Rheum 2010;62:2569-2581.

3. Lard LR, Visser H, Speyer I, et al. Early versus delayed treatment in patients with recent-onset rheumatoid arthritis: comparison of two cohorts who received different treatment strategies. Am J Med 2001;111:446-451.

4. Nell VP, Machold KP, Eberl G, Stamm TA, Uffmann M, Smolen JS. Benefit of very early referral and very early therapy with disease-modifying anti-rheumatic drugs in patients with early rheumatoid arthritis. Rheumatology (Oxford) 2004;43:906-914.

5. Smolen JS, Aletaha D, Bijlsma JW, et al. Treating rheumatoid arthritis to target: recommendations of an international task force. Ann Rheum Dis 2010;69:631-637.

6. Cho SK, Sung YK, Lee H, Bae SC. Validity of estimating EuroQol (EQ5D) from a Health Assessment Questionnaire (HAQ). J Korean Rheum Assoc 2010;17:263-271.

7. Bae SC, Cook EF, Kim SY. Psychometric evaluation of a Korean Health Assessment Questionnaire for clinical research. J Rheumatol 1998;25:1975-1979.

8. Boyd TA, Bonner A, Thorne C, et al. The relationship between function and disease activity as measured by the HAQ and DAS28 varies over time and by rheumatoid factor status in early inflammatory arthritis (EIA): results from the CATCH cohort. Open Rheumatol J 2013;7:58-63.

9. Descalzo MA, Carbonell J, Gonzalez-Alvaro I, et al. Effectiveness of a clinical practice intervention in early rheumatoid arthritis. Arthritis Care Res (Hoboken) 2012;64:321-330.

10. Gremese E, Salaffi F, Bosello SL, et al. Very early rheumatoid arthritis as a predictor of remission: a multicentre real life prospective study. Ann Rheum Dis 2013;72:858-862.

11. Kim D, Choi CB, Lee J, et al. Impact of early diagnosis on functional disability in rheumatoid arthritis. Korean J Intern Med 2017;32:738-746. 